\title{
The role of surgery in coronary artery disease
}

\author{
W. P. Cleland \\ F.R.C.P., F.R.C.S. \\ Department of Surgery, Cardiothoracic Institute, University of London
}

THE role that surgery has to play in the prevention of coronary heart disease is probably negligible but its role in the relief of its dominant symptom, namely angina, is unquestioned. Additionally, it appears likely that a successful coronary artery graft will protect the receipient from the major effects of a subsequent occlusion.

The operation has been extensively performed in the U.S.A. where many thousands of procedures are carried out annually. In the U.K. and in Europe generally there has been a less spectacular explosion. There are figures from some seventeen centres in England involved in coronary artery surgery. In 1971, 200 operations were recorded; in 1972, 300; in 1973, 600; and in 1974, 725.

There has been a steady increase over the last 4 or 5 years, but it should be noted that in three of the seventeen centres over 100 operations were being performed so that these three centres alone accounted for nearly half the operations in the whole of the country. Considering the frequency of angina and the efficacy of surgery in relieving this dominant symptom, it is surprising that the operation has not become much more popular than it has.

In essence, the indications for operation are the presence of angina which cannot be adequately controlled by medical means, where coronary arteriography has demonstrated a severe or total occlusion of a coronary artery with a healthy vessel distal to the obstruction. The condition of the left ventricle plays an important role in assessing the risks of operation.

\section{Pre-operative preparation}

All patients with severe angina being considered for surgery are investigated with coronary arteriography and left ventricular angiography. Those with suitable vessels are then considered for surgery. After the patient has been accepted for operation, it is the practice to correct any of the well known or even less well known risk factors, and it is suggested that these should be adequately corrected by the time the patient is admitted for surgery.

The operation itself is done with the help of cardiopulmonary by-pass. Healthy saphenous veins in the leg are highly desirable, but in their absence the surgeon can employ the rather thin and small $\vec{\circ}$ cephalic vein from the arm or the internal mammary artery. As many anastomoses as are required to by- $\vec{\omega}$ pass the major obstructions are carried out with $\frac{\rho}{\partial}$ autologous vein stretching between the aorta and the coronary artery. The technique of anastomosis is well standardized and the suture material employed by most surgeons is Prolene.

In the post-operative period, arrhythmias may cause some anxiety but these can usually be suppressed with intravenous lignocaine. Post-operative bleeding is the only other occasionally troublesome complication. On the whole, the post-operative course of these patients is remarkably smooth, and it is interesting that the operative mortality is remarkably low.

In the follow-up period, satisfactory results with complete or considerable relief of angina have bee⿳亠丷厂 recorded in numbers varying from $75 \%$ to $90 \%$ Good relief of angina seems to be directly related to the patency of the graft. Approximately $80 \%$ of grafts are patent at the time of coronary arteriography at 6-12 months after operation. Thereafter, there appears to be a small but definite incidence of graft occlusion, possibly approaching $10 \%$ per annum.

Some of the surgical results will now be discussed in a little more detail:

\section{(1) Mortality}

It is very surprising that operations on the coronary arteries carry such a low mortality. Mortality seems to be closely related to the condition of the left ventricle. Thus, if there is good left ventricular function, the operative mortality is between 2 and $4 \%$. If there is slight to moderate left ventricular dysfunction as seen on the angiogram, then the operative mortality will be between 6 and $8 \%$, whilst if there is fairly severe left ventricular dysfunction the operative mortality is between 10 and $12 \%$. The addition of other forms of major surgery, such as the resection of a left ventricular aneurysm, replacement of an aortic valve or replacement of a mitral valve, adds considerably to the operative mortality and figures for these combined operations approach $20 \%$. It should be emphasized that patients in the 
higher risk brackets need not necessarily be excluded from surgery as their need of surgical help is much greater. However, it should be clearly understood by all concerned that the risks will also be greater.

\section{(2) Patency of the graft}

It has been clearly demonstrated that if the grafts remain patent and if grafting has resulted in relief of angina then the symptomatic relief will persist, but that if one or more of the grafts become occluded then angina may recur. What are the factors then which determine graft patency?

(a) Operative techniques. These are clearly important, particularly for the less experienced operator, but the techniques are so standardized that they are unlikely to play a major role in an experienced clinic. Clearly the anastomotic sites must be of adequate size and not rough, irregular or kinked. The graft must travel smoothly from the aorta to the coronary artery without any twists or bends.

(b) The condition of the native coronary artery. This is probably the most important single factor. If the coronary artery is small (less than $1.5 \mathrm{~mm}$ in diameter) or has patches of atheroma, and if the distal coronary bed is diseased so that there is not a good 'run-off', then it is likely that the graft will occlude. Conversely, a coronary artery of $2 \mathrm{~mm}$ or more in diameter which is healthy and where there is a good run-off will almost certainly remain patent. The best evidence of run-off can be obtained by determining flow measurements along the graft at the time of operation. If these are less than about $30 \mathrm{ml} / \mathrm{min}$, the run-off is poor and occlusion is likely. On the contrary, if the flow rate is $\mathbf{9 0}$ or more $\mathrm{ml} / \mathrm{min}$ then the run-off is good and occlusion is much less likely.

(c) Changes in the graft. An autogenous saphenous vein deprived of its natural blood supply and exposed to arterialized blood at systemic arterial pressure undergoes changes of intimal thickening and medial fibrosis which if excessive may lead to an occlusion. Such changes may be aggravated by mishandling of the graft; the latter should always be treated with care.

\section{Long term results}

These are as yet unknown. A successful graft does not appear to affect the natural evolution of atheroma in the native coronary circulation. It is hoped that rigid control of risk factors may delay such changes or prevent new lesions from appearing but as yet there is no proof that this is so.

Coronary grafting is remarkably successful at relieving angina and probably improves the life expectancy of the patient. 